Submission ID: 43805

\title{
Productivity Parameters Prediction on Maps Using Neural Networks
}

S.V. Egorov* (MIMGO), I.I. Priezzhev (IPLAB LLC), A.E. Schelkunov (OOO Pyrgeophisika), E.A. Gladkov (OOO Nord-Imperial, FGBOY VPO NI TPY)

\section{SUMMARY}

The presentation shows the technique and examples for predicting the oil and gas productivity parameters on the map on the basis of a deep neural network with hybrid training and Tikhonov regularization. The results of predicting the effective thickness in continental facies of Western Siberia are shown. The results of comparison between prediction maps obtained by the neural network technique and multidimensional regression are also shown. The advantages of a neural network are efficiency, higher resolution and better correlation coefficient with well data. 


\section{Построение прогнозных карт с помощью нейронных сетей}

С.В. Егоров* (ЗАО «МИМГО»), И.И. Приезжев (ООО «Лаборатория Приезжева»), А.Е. Щелкунов (ООО «Пургеофизика»), Е.А. Гладков (ООО «Норд Империал», ФГБОУ ВПО НИ «ППУ»)

\section{Введение}

Задача прогноза геологического потенциала содержания углеводородов изучаемых геологических слоев (sweet spot analysis) с целью выбора места заложения новых скважин является особенно актуальной и очень сложной в юрских отложениях Западной Сибири и прилегающих регионах. Такая сложность обусловлена сильной латеральной изменчивостью фильтрационно-емкостных свойств (дельтовые, флювиальные фации и т.д.) и приуроченностью юрских отражающих горизонтов (васюганская свита, верхи тюменской свиты) к акустически высоко контрастным, и экранирующим баженовским отложениям. Также иногда имеются контрастные угле содержащие слои.

Прогноз ФЕС в рассматриваемом интервале разреза обычно выполняется с помощью классического атрибутного анализа по картам сейсмических атрибутов на основе построения множественной линейной регрессии (Russell и др., 1997; Leiphartand и др., 2001). Для такого анализа рекомендуется использовать не более 7-8 сейсмических атрибутов максимально некоррелированными между собой. Такое ограничение связано с необходимостью решения системы линейных уравнений и если атрибутов очень много и некоторые из них сильно коррелирует между собой, то решение становится нестабильным, так как главный детерминант системы уравнений будет стремиться к нулю. Выбор используемых в регрессии сейсмических атрибутов выполняется путем простого перебора, и критерием хорошего набора служит коэффициент корреляции между рассчитанным прогнозным параметром и тем же параметром, измеренным в скважинах. Такой способ имеет существенный недостаток, заключающийся в субъективной, рутинной и трудоемкой работе по перебору сочетаний различных сейсмических атрибутов, общее количество которых в настоящее время стремится к бесконечности.

Мы предлагаем использование глубоких нейронных сетей в качестве основного алгоритма атрибутного анализа. Высокая степень свободы нелинейного прогнозного оператора на основе нейронных сетей (Hampson и др., 2001) может дать более точный прогноз с более высоким корреляционным коэффициентом. Также метод менее чувствителен к количеству используемых атрибутов и может работать непосредственно с объёмным распределение исходного сейсмического поля вокруг прогнозируемых точек.

\section{Метод}

Нейронные сети, или более точное название «искусственные нейронные сети», являются попыткой разработки математического аналога биологических нейронных сетей как сетей нервных клеток с основным элементом в виде клеточного нейрона. Основным элементом искусственных нейронных сетей является математический нейрон как многовходовый нелинейный сумматор с одним выходом. Нейронные сети без обратных связей можно представить в виде многослойной конструкции - многослойный персептрон, как показано на рисунке 1. Все нейроны организованы в скрытых слоях между входным и выходным сигналом. Количество выходных нейронов соответствует числу выходов нейронной сети.

Набор векторов (обучающая выборка) подается нейронам на вход в первом скрытом слое, затем преобразованные данные переходят на следующий скрытый слой и так до тех пор, пока сигнал не поступит на выходной нейрон. 


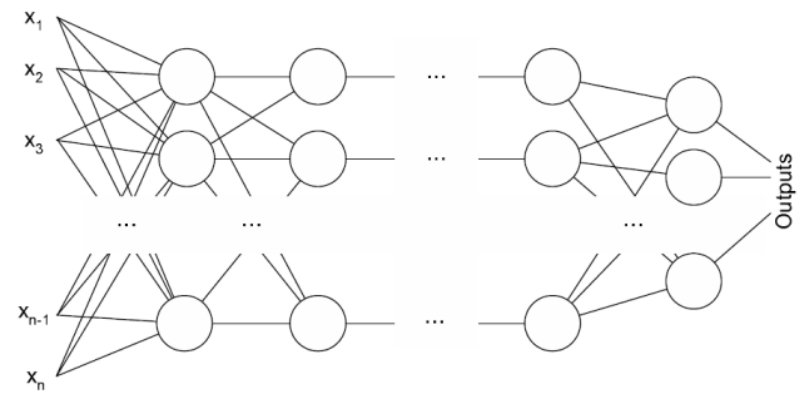

Рисунок 1 Многослойный персептрон.

Для обучения нейронных сетей мы используем гибридные алгоритмы обучения (Kobrunov A., I. Priezzhev, 2015, Kobrunov A., I. Priezzhev, 2016), основные на сочетании методов генерализации первых слоев для глубоких сетей, генетических и градиентных алгоритмов методов оптимизации решений. Основная идея предлагаемой технологии - комбинировать стохастические и детерминированные подходы при построении прогнозирующего оператора на этапе обучения. Предлагаемая технология позволяет избежать многих недостатков генетического алгоритма и градиентных методов, таких как высокая зависимость от начальных данных, явление переобучения, что приводит к созданию оператора с нестабильным предсказанием и низкой скоростью уменьшения ошибки в процессе обучения, и, как результат, низкий уровень качества прогноза. Однако, комбинация этих методов, использует преимущества обоих методов и позволяет находить решение значительно ближе к глобальному минимуму целевой функции по сравнению с простыми градиентными методами, такими как обратное распространение ошибки.

В общем случае, задачу обучения нейронных сетей можно отнести к типичным некорректным задачам - многозначности и неустойчивости решений, а также к возможному отсутствия решения. Например, если количество обучающих пар меньше чем количество неизвестных весовых коэффициентов, то возникает возможность многозначности такого решения. Если обучающие пары имеют сильную корреляцию и все обучающие пары могут быть вычислены через другие, путем линейных операций, то может вообще не существовать такой нейронной сети для такого обучающего множества. Поэтому мы рекомендуем всегда применять методы регуляризации по Тихонову (Тихонов А.Н., Арсенин В.Я., 1987), специально предназначенных для таких задач. В общем случае регуляризация по А.Н. Тихонову представляется в виде добавления к объектной функции дополнительного члена - суммы квадратов всех весовых коэффициентов как показано в формуле 1 (для одного выхода нейронной сети). Коэффициент регуляризации альфа определяет степень «гладкости», что не позволяет быть весовым коэффициентам слишком большими, и это стабилизирует решение.

$$
F=\sum_{k}^{M}\left(y_{k}-y_{k}^{r}\right)^{2}+\alpha \sum_{i}^{K} w_{i}^{2} \rightarrow \min
$$

Где $\mathrm{F}$ - объектная функция для минимизации,

М - количество обучающих пар,

$y_{k}$ - выходное значение в $\mathrm{k}$ - ой обучающей паре,

$y^{r}{ }_{k}$ - вычисленное значение в k- ой обучающей паре

$\mathrm{K}$ - количество неизвестных весовых коэффициентов $w_{i}$,

$\alpha-$ коэффициент регуляризации Тихонова.

\section{Пример}

В качестве примера практического использования данной технологии, приведены карты эффективных толщин разновозрастных пластов Западной Сибири (континентальные отложения), полученные с использованием трехмерного массива сейсмических данных и скважинной информации (рисунок 2-3). Так же, в целях сравнения, приведены результаты прогноза эффективных толщин на основе многомерной регрессии и с использованием нейронных сетей (рисунок 3). Все результирующие прогнозные карты демонстрирует полное соответствие геологическим представлениям о строении изучаемых пластов. 


\section{EAGE}

EUROPEAN

ASSOCIATION OF

ENGINEERS

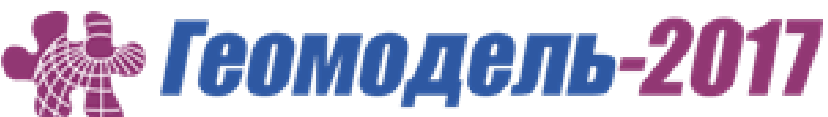

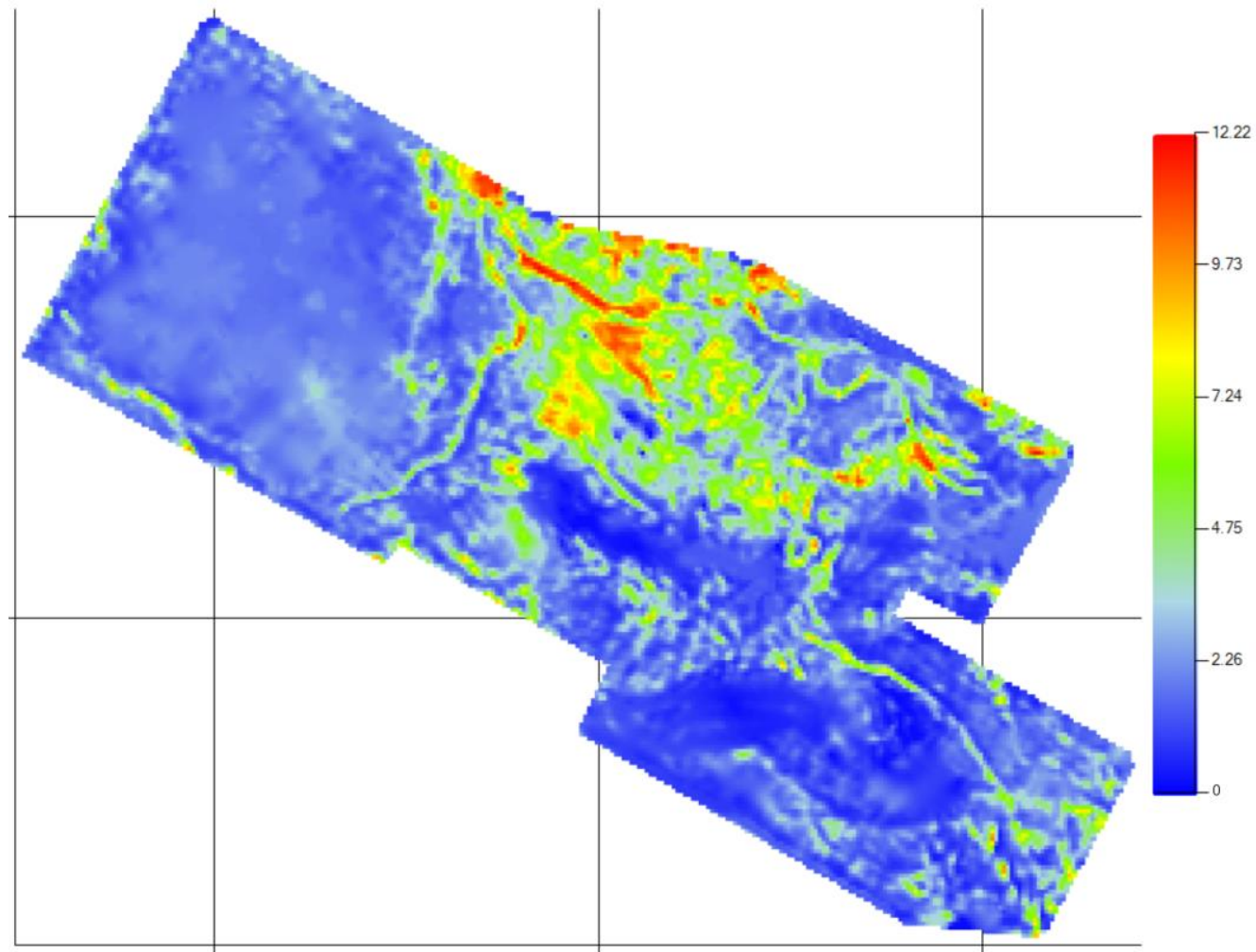

Рисунок 2 Прогнозная карта эффективных толщин в юрских отложения

1)

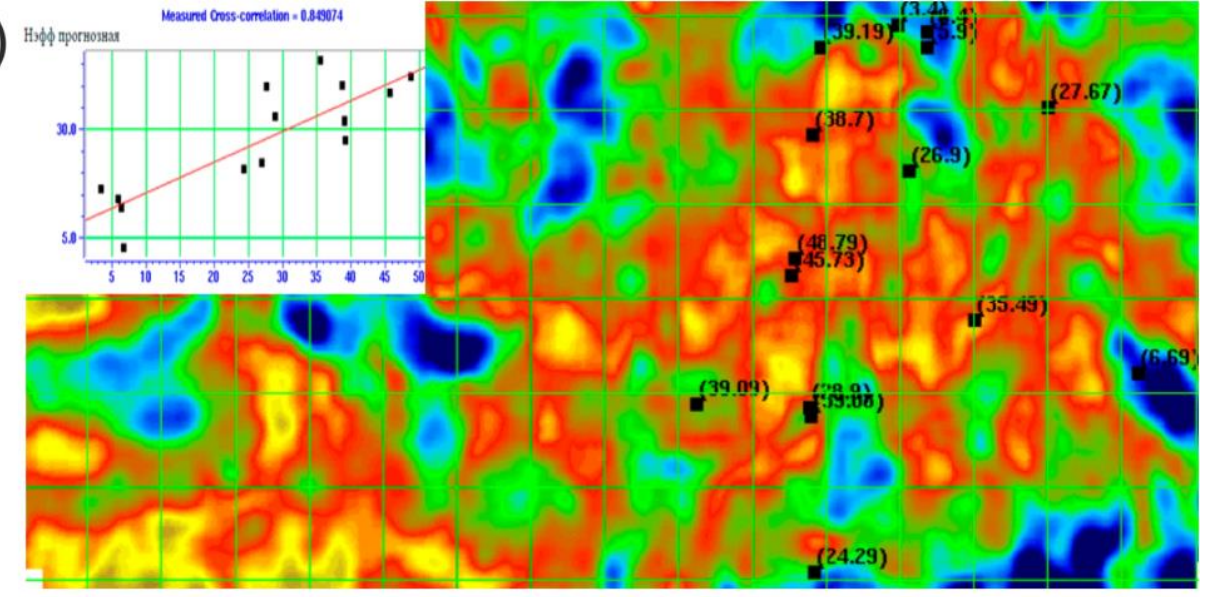

2)

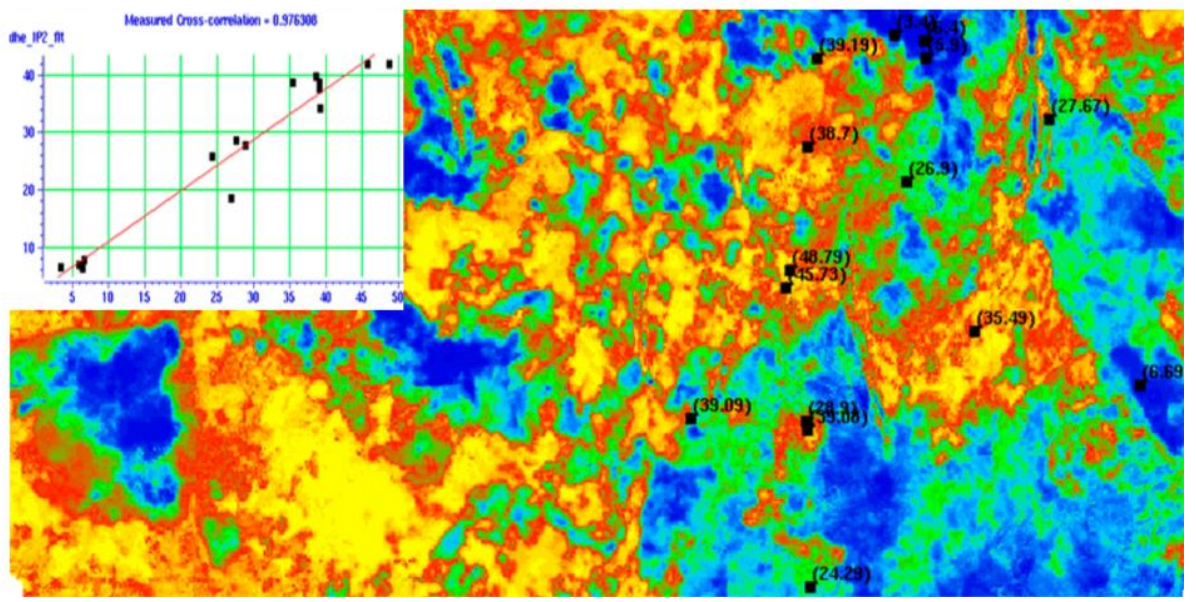

Рисунок 3 1) Карта эфф. толщин полученная многомерной регрессией 2) Карта эфф. толщин полученная через нейронные сети 


\section{Выводы}

Использование глубоких нейронных сетей позволяет выполнять прогнозные построения в сложных геологических условиях, в том числе с высокой степенью латеральной изменчивости и со слабым сейсмическим откликом. В свою очередь, сравнение прогнозных карт, полученных различными методами, показывает очевидное преимущество применения алгоритмов на основе нейронных сетей, заключающееся в более детальном, точном и оперативном результате прогноза. Преимущества рассматриваемой методики достигаются путем применения нелинейного прогнозного оператора с высокой степенью свободы и возможностью использования скользящего окна в пространстве.

\section{Список литературы на русском языке}

Тихонов А.Н., Арсенин В.Я. Методы решения некорректных задач. М.: Наука. Главная редакция физико-математической литературы, 1979. Изд. 2-е. - 288 с.

Hampson, B., J. Schuelke, and J. Quirein, 2001, Use of multi-attribute transforms to predict $\log$ properties from seismic data: Geophysics, 66, no. 1, 3-46.

Kobrunov A., I. Priezzhev, 2015, Stable Nonlinear Predictive Operator Based on Neural Network, Genetic Algorithm and Controlled Gradient Method, New Orleans, 2015 SEG Annual Meeting.

Kobrunov A., I. Priezzhev, 2016, Hybrid combination genetic algorithm and controlled gradient method to train a neural network, GEOPHYSICS, VOL. 81, NO. 4, 1-9.

Leiphartand D.J. and B. S. Hart, 2001, Comparison of linear regression and a probabilistic neural network to predict porosity from 3-D seismic attributes in Lower Brushy Canyon channeled sandstones, southeast New Mexico, Geophysics, 66, no. 5, 1349-1358.

Priezzhev I.I., L.E. Shmaryan, G. Bejarano, 2008, Nonlinear multitrace seismic inversion using neural network and genetic algorithm, - 3rd EAGE St. Petersburg International Conference.

Russell, B., D. Hampson, J. Schuelke, and J. Quirein, 1997, Multi-attribute seismic analysis: The Leading Edge, 16, no. 10, 1439-1443.

\section{References}

Tihonov A.N., Arsenin V.Ya. Metodyi resheniya nekorrektnyih zadach. M.: Nauka. Glavnaya redaktsiya fiziko-matematicheskoy literaturyi, 1979. Izd. 2-e. - $288 \mathrm{~s}$.

Hampson, B., J. Schuelke, and J. Quirein, 2001, Use of multi-attribute transforms to predict log properties from seismic data: Geophysics, 66, no. 1, 3-46.

Kobrunov A., I. Priezzhev, 2015, Stable Nonlinear Predictive Operator Based on Neural Network, Genetic Algorithm and Controlled Gradient Method, New Orleans, 2015 SEG Annual Meeting.

Kobrunov A., I. Priezzhev, 2016, Hybrid combination genetic algorithm and controlled gradient method to train a neural network, GEOPHYSICS, VOL. 81, NO. 4, 1-9.

Leiphartand D.J. and B. S. Hart, 2001, Comparison of linear regression and a probabilistic neural network to predict porosity from 3-D seismic attributes in Lower Brushy Canyon channeled sandstones, southeast New Mexico, Geophysics, 66, no. 5, 1349-1358.

Priezzhev I.I., L.E. Shmaryan, G. Bejarano, 2008, Nonlinear multitrace seismic inversion using neural network and genetic algorithm, - 3rd EAGE St. Petersburg International Conference.

Russell, B., D. Hampson, J. Schuelke, and J. Quirein, 1997, Multi-attribute seismic analysis: The Leading Edge, 16, no. 10, 1439-1443. 